\title{
nature
}

photonics

\section{Shrinking down}

\author{
The ability to make ever smaller and more sophisticated optical circuits for controlling light is \\ now moving into a new dimension, below the wavelength of light.
}

One of the big challenges facing photonics is undoubtedly the creation of sophisticated miniature optical circuits that can store, route and process digital information on a subwavelength scale (that is, hundreds of nanometres). Once realized such photonic very-large-scale-integration (VLSI) circuits could be at the heart of future computers and telecommunication systems.

These optical chips could relieve the burden from electronics as issues such as heat generation, bandwidth, transmission losses and size all become increasingly problematic in electronic devices as they get smaller and the packing density increases.

The astonishing progress reported over the past few years in the fields of silicon photonics, photonic crystals, plasmonics and other technologies is a cause for optimism that the challenge can be met. Indeed, three articles in this issue indicate that the momentum is being maintained.

First, it may transpire that an approach based on structural phase changes in nanoparticles could be an elegant answer to making nanoscale optical switches, memories and modulators according to this month's commentary. On page 551, Nikolay Zheludev describes how phase changes on the nanoscale could potentially be triggered by just a handful of photons. On a larger scale, structural phase changes, such as ice turning to water, are a commonly observed and well-understood phenomenon. The study of such changes in the nanoworld and their application to photonics could turn out to be a fruitful area of research.

Second, on page 589, Hill and coworkers from the COBRA Research Institute in the Netherlands and the Korea Advanced Institute of Science and Technology (KAIST) report an electrically pumped nanocavity laser that is made by coating a semiconductor heterostructure with a thin gold film. Although the demonstrated device operates at cryogenic temperatures, its small size, low threshold current and potential for high-speed operation make it a promising candidate for photonic VLSI. The news is significant as it was previously thought that metal-based cavities would be far too lossy to create practical nanolasers, but it now appears that this is not the case. The next challenge is to shrink the lasers further from several hundreds to a few tens of nanometres and raise the temperature of operation.

Third, when it comes to manipulating light on a subwavelength scale, surface plasmon polaritons (SPPs) are a prime candidate and have already been shown to be capable of guiding and modulating light. On page 573, Kuipers et al. from the FOM Institute for Atomic and Molecular Physics in the Netherlands describe how specially designed structures can also control the effective speed of propagation of SPPs. In this particular demonstration, the group velocity of femtosecond SPP wavepackets was reduced by a factor of two. This ability to control the speed of such SPPs could ultimately lead to highly compact delay lines or low-power all-optical switches. Although the devices are on the microscale rather than the nanoscale at present, it is a great example of what plasmonics has to offer.

\section{A unique event}

To celebrate the launch of Nature Photonics, a three-day conference that will discuss the future evolution of optical communication, is being held in Japan this month. The event, formally called "The Nature Photonics Technology Conference - Optical Communications" will take place in Tokyo from 23 to 25 October.

The idea behind the event is to create a unique platform for a stimulating and interactive discussion of what the future holds for optical communications. Around
20 speakers from around the world, each a well-regarded expert or an influential figure within industry, have been invited to share their perspectives on the topic.

Coverage will span the whole remit of optical communication with talks ranging from next-generation component and device technologies through to systems and transmission techniques and digital services. As with Nature Photonics, the event is intended to have broad appeal and be of interest to everyone working in photonics.
In addition to a packed program of scheduled talks there will be a panel discussion on the theme of the big challenges facing photonics and a chance to meet the editorial team of Nature Photonics.

Please do come along if you have the opportunity. To find out more about the event, including a speaker list, program and registration information please visit http://www.nature.com/natureconferences/ photonics/index.htm 